\title{
About the linearity of the color-magnitude relation of early-type galaxies in the Virgo cluster
}

\author{
Analía V. Smith Castelli ${ }^{1}$ \\ Instituto de Astrofísica de La Plata (CCT-La Plata, CONICET-UNLP), Paseo del Bosque s/n, \\ B1900FWA, La Plata, Argentina \\ asmith@f caglp.unlp.edu.ar \\ Nélida M. González and Favio R. Faifer ${ }^{2}$ \\ Facultad de Ciencias Astronómicas y Geofísicas, Universidad Nacional de La Plata, Paseo del Bosque s/n, \\ La Plata, B1900FWA, Argentina \\ ngonzalez@fcaglp.unlp.edu.ar,favio@f caglp.unlp.edu.ar \\ Juan Carlos Forte \\ CONICET - Planetario de la Ciudad de Buenos Aires "Galileo Galilei", Av. Sarmiento y B. Roldán, \\ Ciudad Autónoma de Buenos Aires, Argentina \\ forte@f caglp.unlp.edu.ar
}

\begin{abstract}
We revisit the color-magnitude relation (CMR) of the Virgo cluster early-type galaxies in order to explore its alleged non-linearity. To this aim, we reanalyze the relation already published from data obtained within the ACS Virgo Cluster Survey of the Hubble Space Telescope, and perform our own photometry and analysis of the images of the 100 early-type galaxies observed as part of this survey. In addition, we compare our results with those reported in the literature from data of the Sloan Digital Sky Survey. We have found that when the brightest galaxies and untypical systems are excluded from the sample, a linear relation arises in agreement with what is observed in other groups and clusters. The central regions of the brightest galaxies also follow this relation. In addition, we notice that Virgo contains at least four compact elliptical galaxies besides the well known object VCC 1297 (NGC 4486B). Their locations in the $\left\langle\mu_{\mathrm{eff}}\right\rangle-$ luminosity diagram define a different trend to that followed by normal early-type dwarf galaxies, setting an upper limit in effective surface brightness and a lower limit in effective radius for their luminosities. Based on the distribution of different galaxy sub-samples in the color-magnitude and $\left\langle\mu_{\text {eff }}\right\rangle$-luminosity diagrams we draw some conclusions on their formation and the history of their evolution.
\end{abstract}

Subject headings: galaxies: clusters: general-galaxies: clusters: individual (Virgo) — galaxies: dwarfgalaxies: elliptical and lenticular, $\mathrm{cD}$ - galaxies: photometry

\footnotetext{
${ }^{1}$ Facultad de Ciencias Astronómicas y Geofísicas, Universidad Nacional de La Plata, Paseo del Bosque s/n, La Plata, B1900FWA, Argentina

${ }^{2}$ Instituto de Astrofísica de La Plata (CCT-La Plata, CONICET-UNLP), Paseo del Bosque s/n, B1900FWA, La
}

\section{Introduction}

The color-magnitude relation (CMR) of earlytype galaxies is a well known photometric relation

Plata, Argentina 
that has been studied for long (e.g. Baum 1959. Visvanathan \& Sandage 1977; Secker et al. 1997; Terlevich et al. 2001: López-Cruz et al. 2004: Andreon et al. 2006: Smith Castelli et al. 2008a: Misgeld et al. 2008. 2009; Janz \& Lisken 2009; Smith Castelli et al. 2012, Lieder et al. 2012). In the color-magnitude diagram (CMD), early-type galaxies define a sequence in which giant galaxies are redder than dwarfs. Spectroscopic and infrared photometric studies of early-type galaxies have shown that this relation is mainly driven by differences in metal abundances (e.g. Faben 1973: Terlevich et al. 1999; Trager et al. 2000; Clemens et al. 2011).

It is widely accepted that when optical photometric bands are considered, the CMR is linear along its extension, and that it shows no perceptible change of slope from the bright galaxies to the dwarf regime except, sometimes, at the very bright end (e.g. Secker et al. 1997, $(B-R)$ vs. $R$ in Coma: Terlevich et al. 2001, $(U-V)$ vs. $V$ in Coma; López-Cruz et al. 2004, $(B-R)$ vs. $R$ in Coma and 57 X-ray detected clusters; Andreon et al. 2006, $(B-V)$ vs. $R$ and $(V-R)$ vs. $R$ in Abell 1158; Smith Castelli et al. 2008a, 2012. $\left(C-T_{1}\right)$ vs. $T_{1}$ in Antlia; Misgeld et al. 2008. $(V-I)$ vs. $V$ in Hydra; Misgeld et al. 2009, $(V-I)$ vs. $V$ in Centaurus). In addition, there is strong evidence for the universality of this relation in clusters of galaxies which led several authors to suggest its use as a reliable indicator of distance and cluster membership (e.g. Sandage 1972; Binggeli \& Jerien 1998; López-Cruz et al. 2004; Chiboucas et al. 2010).

Despite all the evidence about the existence of a common linear CMR in nearby groups and clusters, non-linear trends have been reported in Virgo. Janz \& Lisken (2009) and Chen et al. 2010, hereafter, Ch10) have obtained an inverse S-shaped relation that seems to be consistent with the quadratic fit performed by Ferrarese et al. 2006, hereafter, F06). More recently, Lieder et al. 2012) have reported a linear relation that changes its slope at $M_{V} \approx-14 \mathrm{mag}$, where the dSph regime begins.

Based an all these findings, the CMR of Virgo early-type galaxies and, therefore, Virgo's earlytype galaxy population itself, would display distinct characteristics in comparison with similar populations in other groups and clusters. These characteristics should be explained in the light of theoretical models or/and observational data.

Bearing this in mind, we revisit Virgo's CMR by performing our own photometry and analysis on the images of 100 early-type galaxies included in the ACS Virgo Cluster Survey (ACSVCS) of the Hubble Space Telescope (HST). This material has been already used by F06 to analyse this relation. Here we present a new analysis based on photometric parameters obtained with a simpler approach than that followed by F06. Basically, we obtained brightness profiles by fitting elliptical isophotes with fixed parameters, and calculated isophotal limited magnitudes, colors and effective radii from these brightness profiles. By comparing our results with those of F06 and those obtained by Ch10 from data of the Sloan Digital Sky Survey $(S D S S)$, we aim to explore the alleged nonlinearity of the relation.

The paper is organized as follows. Section2 presents the data and our approach to obtain photometric and structural parameters. Section 3 shows our analysis of the color-magnitude and mean effective surface brightness $\left(\left\langle\mu_{\text {eff }}\right\rangle\right)$ luminosity diagrams obtained from the photometry of F06. We also compare these diagrams with those built from our photometry and from the photometry of Ch10. Section 4 presents a discussion of the results, and Section 5 our conclusions.

\section{Observations and photometry}

From the Multimission Archive at the Space Telescope Science Institute (MAST), we downloaded the images of 100 early-type galaxies confirmed as members of the Virgo cluster through radial velocities. These images were obtained in the framework of the ACSVCS Côté et al.2004), and were taken with the F475W and F850LP filters and automatically processed with the CALACS and MultiDrizzle packages Koekemoer et al.2002). Short exposures images were obtained in order to recover the very central regions of galaxies with saturated nuclei. However, we decided to work only with those of long exposures $(750 \mathrm{sec}$. for the F475W filter and $1200 \mathrm{sec}$. for the F850LP one) as the overexposed zones represent just the inner $0.4^{\prime \prime}$ in the worst cases. The field of view (FOV) of the ACS camera is $202 \times 202^{\prime \prime}$ and subtends a spatial scale of $0.049^{\prime \prime}$ pixel $^{-1}$. Following 
F06, we will adopt a distance modulus for Virgo of $(m-M)=31.09$, which translates into a distance of $D=16.6 \mathrm{Mpc}$. At this distance, $1^{\prime \prime}$ subtends $\sim 80 \mathrm{pc}$.

Our photometry, presented in Table1 was obtained with ELLIPSE within IRAF, adopting a simpler approach than that followed by F06. As a first step, we constructed a master mask to fill the gaps and cover the bad pixels of the ACS CCDs. After that, we built individual masks for each galaxy in order to avoid bright or saturated objects and bad or overexposed regions. From the galaxies images in the $\mathrm{F} 475 \mathrm{~W}$ filter, we obtained their center coordinates, and from the (F475WF850LP) color maps, we estimated their global ellipticities and position angles as those shown by their external regions. Then, we fitted elliptical isophotes keeping these parameters fixed during the run.

Eight galaxies displayed saturated central regions. In these cases, the isophotes were fitted excluding these regions, from an initial radius of $0.4^{\prime \prime}$ at most. All of them are bright objects $\left(M_{g} \lesssim-18.9 \mathrm{mag}\right)$, so we do not expect that the excluded regions significantly affect the integrated colors and luminosities.

Once we obtained the brightness profiles in both filters, we examined the images one by one in order to identify those galaxies that are not fully contained in the FOV of the ACS (hereafter, incomplete galaxies). For those galaxies that are complete in the ACS frames (hereafter, complete galaxies), we corrected the sky level by constructing growth curves, i.e., plots of integrated flux versus semi-major axis. The correction to the sky level is the value for which these curves display an asymptotic flat behavior to infinity.

After that, we calibrated all brightness profiles to the ABMAG system Sirianni et al. 2005) following F06. Through this calibration, F475W and F850LP surface brightnesses transform into $g_{A B}$ and $z_{A B}$ ones, respectively, which resembles the SDSS $g$ and $z$ filters.

Integrated SDSS $g$ and $z$ magnitudes, and the corresponding $(g-z)$ colors, were obtained by integrating the calibrated brightness profiles in each filter. For complete galaxies, the integration was performed up to $\mu_{g_{0}} \approx 27 \mathrm{mag} \operatorname{arcsec}^{-2}$. For incomplete ones, the integration was performed up to a limiting surface brightness of $\mu_{g_{0}} \approx 24 \mathrm{mag}$ $\operatorname{arcsec}^{-2}$, as the corresponding isophotes did not reach the edge of the images. We expect that at this brightness the profiles are not strongly affected by the adopted sky value: for complete galaxies, the sky correction at this level is $\lesssim 0.12$ mag $\operatorname{arcsec}^{-2}$. Regarding the effect of the different surface brightness limits on the integrated magnitudes, we find an average difference for complete galaxies of $\left\langle\left(g_{24}-g_{27}\right)\right\rangle=1.0 \pm 0.8 \mathrm{mag}$.

Finally, magnitudes and colors, as well as brightness and color profiles, were extinction and reddening corrected considering $A_{g}=3.591 E(B-$ $V)$ and $A_{z}=1.472 E(B-V)$ Sirianni et al. 2005). The values of $E(B-V)$ for each galaxy were obtained from table 1 of F06.

We also estimated effective radii $\left(r_{\text {eff }}\right)$ for the complete galaxies. To do this, we considered the total flux of the galaxy as that contained within the limiting isophote used to obtain magnitudes and colors, and the $r_{\text {eff }}$ as the equivalent radius that contains half of that flux. From these values, we calculated the corresponding $\left\langle\mu_{\text {eff }}\right\rangle$ following equation 1 of Smith Castelli et al. 2008a . In this way, our $r_{\mathrm{eff}}$ and $\mu_{\mathrm{eff}}$ values are model independent. In addition, it should be noticed that they will probably reflect the effects analyzed by Trujillo et al. 2001) related with structural parameters obtained from images limited in their radial extent (for example, it is expected that the $r_{\text {eff }}$ of galaxies with $n \geq 4$ are underestimated by a factor of 2 , and their $\left\langle\mu_{\text {eff }}\right\rangle$, overestimated in 1 mag or more).

In the publicly available webpage of our groun 1 we show the $F 475 W$ images, $(g-z)$ color maps, surface brightness profiles and color profiles obtained from our photometry for the 100 galaxies of the ACSVCS. They are grouped according to the zone that occupy in the CMD (see Section 3.1), ordered by their VCC number.

\section{Results}

\subsection{Virgo CMR built from the ACS pho- tometry of F06}

In the top panel of Figure 1 we show the CMD of the galaxies observed within the ACSVCS. To build this plot, we used the extinction and red-

\footnotetext{
${ }^{1}$ http://fcaglp.unlp.edu.ar/CGGE/Virgo/Virgolist.html
} 
dening corrected $g$ magnitudes and $(g-z)$ colors presented by F06 in their table 4 . We recall here that these magnitudes exclude the nucleus and are obtained by integrating the best-fit profile to infinity, while colors are measured directly from the surface brightness profile in the range $1^{\prime \prime}<r<r_{\text {eff }}$. Absolute magnitudes are calculated from the distance moduli of the individual galaxies given by Mei et al. 2007) when available. We exclude from the sample the galaxies VCC 538, VCC 571, VCC 575, VCC 731 and VCC 1025 as Mei et al. 2007) found they lie behind the Virgo cluster.

In the CMD we can distinguish three regions:

1. A break at the bright end defined by VCC 763, VCC 798, VCC 881, VCC 1226, VCC 1316, VCC 1632, VCC 1903 and VCC 1978.

There is a gap between this break and the rest of the galaxies, at $M_{g_{0}} \sim-20.6 \mathrm{mag}$.

2. A red group depicted by VCC 1192, VCC 1199 , VCC 1297, VCC 1627 and VCC 1871.

3. A blue zone defined by VCC 21 , VCC 1488 , VCC 1499, VCC 1779 and VCC 1857.

With the exception of VCC 1327, the rest of the galaxies define what seems to be a linear trend with a gap at $M_{g_{0}} \sim-17.5$ mag (hereafter, intermediate gap). This gap is probably due to an incompleteness effect as the ACSVCS sample is $\sim 60 \%$ complete, while it is $100 \%$ complete for galaxies brighter than $M_{g_{0}} \sim-19.5$ mag (Ch10).

Taking into account the errors given by F06 for $(g-z)$ colors, we performed several fits to the linear trend. In Table2 we show the values obtained for the different parameters. There seems to be a change of slope between the bright $\left(M_{g_{0}} \lesssim-17.5\right)$ and faint $\left(M_{g_{0}}>-17.5\right)$ parts of the linear relation. However, this is not a reliable result as the sub-samples considered to perform these fits display weak linear correlations $(r<-0.6)$. This weakness is also detected in the analysis of the intrinsic scatter of the relation. When the number of galaxies considered to perform the bright and faint fits vary, we obtain contradictory results for $\sigma_{(g-z)_{0}}$ (it increases towards the faint end in the whole sample, while it decreases in the reduced one).
However, it can be stated that the dispersion in the relation might not be due to distance effects. The mean measurement error in the distance moduli reported by Mei et al. 2007) is 0.07 mag. We have found that the intrinsic scatter in magnitude around the mean relation is $0.82 \mathrm{mag}$.

When we build the $\left\langle\mu_{\text {eff }}\right\rangle$-luminosity diagram from the data obtained by F06 (bottom panel of Figure 1), we can distinguish three different zones that deviate from a linear color-magnitude trend. The very bright galaxies defining the bright break in the CMD also define a break from the general trend followed by most of the galaxies. The objects of the red group set a low boundary in $r_{\text {eff }}$ and a high one in $\left\langle\mu_{\text {eff }}\right\rangle$ to the whole sample. The galaxies that depict the linear trend in the CMD are found around the locus of constant $r_{\mathrm{eff}} \sim 1$ kpc, with most of them displaying $0.5<r_{\text {eff }}<2.0$ kpc. However, the galaxies that show the bluest colors are placed within the trend of dwarf earlytype galaxies.

From the images, color maps and brightness profiles of the galaxies, it can be seen that the objects defining the bright break in the CMD are very bright ellipticals and S0s. In particular, this sub-sample includes the three dominant Virgo galaxies VCC 881 (M86), VCC 1226 (M49) and VCC 1316 (M87).

Within the red group, all of the galaxies display characteristics typical of compact elliptical (cE) galaxies. That is, round and compact morphologies, red colors for their luminosities and concentrated brightness profiles $\left(r_{\mu_{z_{0}}=25} \leq 40^{\prime \prime}\right)$ with high central surface brightness $\left(\mu_{z_{0}} \approx 16 \mathrm{mag}\right.$ $\left.\operatorname{arcsec}^{-2}\right)$. In particular, VCC 1297 is the well known cE galaxy of the Virgo cluster, NGC 4486B. Our identification is in agreement with that of Ch10 regarding VCC 1192, VCC 1199, VCC 1297 and VCC 1627 . We add the galaxy VCC 1871.

Most of the galaxies defining the blue zone display blue centers that can be observed both in the color maps and in the color profiles. The exception is VCC 1857 which does not show a significant color gradient but it is quite blue $\left((g-z)_{0} \sim 0.9\right.$ mag). Mei et al. 2007) were not able to estimate distance moduli for most of these galaxies through the surface brightness fluctuation method because of their extremely blue colors.

Among the galaxies that define the linear trend, 
we noticed that VCC 1512 is clearly inconsistent with the size and morphology displayed by the rest of the systems of similar integrated magnitudes. F06 have reported $r_{\text {eff }}=497.5^{\prime \prime}$ but they pointed out that all of its integrated quantities should be considered unreliable. In the case of the galaxies that are behind Virgo, a similar situation is found for VCC 575 as F06 have reported $r_{\text {eff }}=490^{\prime \prime}$.

\subsection{Virgo CMR built from our ACS pho- tometry}

In the top panel of Figure2 we show the CMD of the galaxies included in the ACSVCS obtained from our photometry. It is worth noticing that VCC 1327 follow the general trend in this case. As noticed by F06, there is a bright foreground star near the center of the galaxy. To obtain VCC 1327 brightness and color profiles, we masked the star. In addition, we have obtained photometric parameters for VCC 1030. F06 do not give an estimate of magnitudes, colors or $r_{\text {eff }}$ values for this galaxy. Therefore, we include these two objects in our analysis. For VCC 1535, as it is an incomplete galaxy, we obtained low limits for its integrated magnitudes and $(g-z)$ color. F06 have not reported integrated quantities or structural parameters for it.

In spite of these differences and those analyzed in Appendix $\mathrm{A}$ we reproduce the general trend of the CMR. In particular, the linear region with its intermediate gap, as well as the red and blue zones. As expected, we lose the bright break as the galaxies that define it are incomplete ones and the magnitudes obtained from our photometry are underestimated.

In Table2 we show the results of performing different fits to the linear trend excluding incomplete, $\mathrm{cE}$ and blue galaxies. We can see that, as in the case of the F06 CMD, there seems to be a change of slope between the bright and faint end of the trend, although in the opposite way. However, both slopes are comparable within the errors in spite of the weak linear correlation of the sub-samples. In addition, we have found a slight increase of the intrinsic scatter towards the faint end of the relation.

It should be noticed that, in our case, we considered the errors in both variables to perform the different fits, while we could only take into account the errors in $(g-z)$ in the case of F06 as these authors do not provide magnitude errors.

We would like to point out that if we consider exactly the same galaxy sample both for F06 and our photometry (Reduced Sample in Table2), we obtain, within the errors, the same slope and zero point for the relation. These values are also consistent with those obtained for the whole trend in each case. In addition, the apparent change of slope due to the low linear correlation of the samples is also recovered.

Finally, we consider our sample with magnitudes and colors integrated between $1^{\prime \prime}$ and $1 r_{\text {eff }}$, excluding incomplete, $\mathrm{cE}$ and blue galaxies. In this case we obtain:

$(g-z)_{0}=1.37( \pm 0.014)-0.096( \pm 0.007)\left(M_{g_{0}}+18\right)$

with $r=-0.87$ and $\sigma_{(g-z)_{0}}=0.07$. It is interesting to note that the slope of the relation does not significantly change by considering a different radius range to perform color and magnitude integrations, and it is identical within the errors to those found in the photometry of F06. However, the zero point turns out to be redder which is consistent with the fact that, in this case, our magnitudes and colors are measured within $1 r_{\text {eff }}$.

It is remarkable that as a consequence of our measurement procedure, the central regions of the brightest incomplete galaxies show magnitudes and colors that suit so well in the linear trend. We emphasize that these galaxies were not included in the sample considered to perform the fits as their integrated magnitudes were obtained by integrating their brightness profiles up to $\mu_{g_{0}} \approx 24 \mathrm{mag}$ $\operatorname{arcsec}^{-2}$. As can be seen from the color profiles of the galaxies of the bright end, this brightness is reached below $1 r_{\text {eff }}$. Therefore, we find that in the CMD, the central regions of bright early-type galaxies seem to follow the linear trend defined by fainter ones.

We also built the $\left\langle\mu_{\mathrm{eff}}\right\rangle$-luminosity plane with the complete galaxies. We did not include the incomplete ones because we could not estimate their $r_{\text {eff }}$. Despite the differences between our $r_{\text {eff }}$ values and those reported by F06 for the complete

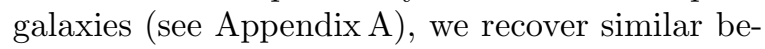
haviors. That is, the galaxies of the red cloud set upper and lower limits in $\left\langle\mu_{\text {eff }}\right\rangle$ and $r_{\text {eff }}$, respectively; the galaxies that define the linear trend 
tend to be located around the locus of constant $r_{\text {eff }} \sim 1 \mathrm{kpc}$; and the bluest galaxies are placed on the trend of typical early-type dwarf galaxies.

\subsection{Virgo CMRs built from the SDSS photometry of Ch10}

To support our results we worked with the SDSS $g_{\mathrm{COG}}$ vs. $(g-z)_{\mathrm{t}}$ photometry of Ch10 and the distance moduli of Mei et al. 2007). We can see from Table2 that the same reduced sample considered above, gives identical parameters to those of F06 and our work, within the errors. Furthermore, if we take the whole ACSVCS sample excluding the bright break, $\mathrm{cE}$ and blue galaxies, but with the photometry of Ch10 (79 objects), we obtain similar results to those found from our photometry.

We also obtain strong linear relations when other SDSS color combinations are considered, as can be seen from the left panel of Figure3 The right panel shows the $\left\langle\mu_{\text {eff }}\right\rangle$-luminosity diagram built with the values presented in table 1 of Ch10. It is similar to that of F06, and is similar to our diagram if we exclude the incomplete galaxies.

\section{Discussion}

\subsection{The bright break of the CMR}

The bright break in the CMR has been observed in, for example, the Hydra cluster. Although Misgeld et al. 2008) have fit a linear relation to the whole sample, a detachment from the general trend in the very bright end is evident from their figures $8 \mathrm{~b}$ and 10 . It can also be marginally detected in the Centaurus cluster, where the break is defined by the two brightest galaxies Misgeld et al. 2009) and even in the Coma CMR obtained by Secker et al. (1997). The break is also seen in the CMRs of the Virgo cluster obtained by Janz \& Lisker 2009) and Ch10 with data from the SDSS. It should be noticed, however, that in the CMR of Ch10, the bright break is only defined by six galaxies (VCC 763, VCC 798, VCC 881, VCC 1226, VCC 1316 and VCC 1978), while that of F06 is defined by eight objects. This discrepancy is probably due to the difficulty of measuring large galaxies in the small FOV of the ACS. In this sense, it is worth noticing the clear differences between the values of the $r_{\text {eff }}$ found by F06 and Ch10 for the galaxies of the bright break.
From the point of view of numerical simulations, Jiménez et al. 2011) have shown that the bright break in the CMR may arise due to the contribution of dry mergers to the final mass of the brightest galaxies of clusters. Dry mergers involve little amount of gas and, therefore, they do not trigger starbursts that can modify the metal content of the galaxies. On the contrary, they only contribute to increase the stellar mass and, therefore, the total luminosity at constant metallicity (i.e. color). The bright break in these simulations is found at $M_{R} \sim M_{V} \sim M_{T_{1}} \approx-20 \mathrm{mag}$. In the CMR of F06, it is observed at $M_{g_{0}} \sim-20.6 \mathrm{mag}$ and in that of Ch10, at $M_{g_{0}} \sim-21.5$ mag.

On the other hand, Hilz et al. 2012, and references therein) have found that stripped material from minor mergers is expected to alter the external regions of the galaxies rather than the inner ones, increasing the size of compact massive spheroidal galaxies similar to those observed at $z \sim 2$. In this context, our findings about the central regions of massive galaxies behaving as lowmass early-type galaxies in the CMD, could be linked with this picture. If the accreted low-mass satellites were metal-poor (thus blue), they would make the global color of the growing galaxies bluer than the linear CMR (see also Hilker et al. 1999; Côté et al. 2000).

It is interesting that the galaxies defining the bright break in the CMR, also detach from the general trend followed by the rest of the galaxies in the three-dimensional plot presented by Forte et al. 2009). This plot represents a logarithmic volume space defined by stellar mass, projected stellar mass density and total GC efficiency of formation. The authors conclude that the different behavior among the brightest galaxies and the rest of the sample may be associated with different merger histories. For these authors, galaxies with masses below $10^{11} M_{\odot}$ might have experienced a relatively low number of dissipationless merging events while systems with masses above that value, might mainly have experienced major mergers.

\subsection{The linear region of the CMR}

From our analysis we have found that complete non-peculiar early-type galaxies with luminosities in the range $-20.5 \lesssim M_{g_{0}} \lesssim-16.5 \mathrm{mag}$, which are confirmed members of the Virgo cluster, define a 
linear trend in the CMD. It is worth noticing that from the ACSVCS sample, it can only be stated that the CMR of Virgo is linear in the above range which covers four magnitudes in brightness. As a comparison, the CMR of the Antlia cluster covers $\sim 10$ magnitudes considering spectroscopically confirmed members Smith Castelli et al. 2012).

The linear region is also recovered in the CMD built from the SDSS photometry of the ACSVCS galaxies of Ch10, as it was shown in Section 3.3 They report an inverted S-shape trend in agreement with Janz \& Lisker 2009). However, this feature arises as an attempt to reproduce the whole trend, including the brightest galaxies, the cEs and those of the blue zone. In particular, the latter galaxies have been considered in previous works as dE/dIrr transition objects which, in our opinion, should be excluded from an early-type galaxy sample.

The analysis of F06 of the relation is quite similar to our work as they do not include most of the galaxies of the red group and the blue zone. However, to fit the whole relation including the very bright end, requires, at least, a second order solution.

Regarding the galaxies that populate the faint end of the CMD, which mostly define the inverted S-shape reported by Janz \& Lisker 2009), a point to take into account is, for example, the different image resolution between the data obtained from HST-ACS and SDSS (a factor of $\sim 15$, Ch10). Bearing this in mind, it cannot be ruled out that the sample used by Janz \& Lisker 2009) includes a considerable amount of background galaxies that would populate the whole CMD (see figure 2 in Smith Castelli et al. 2012 for a visualization of this effect), and dE/dIrr objects placed in the blue side of the early-type relation. All these systems would contribute to depict an S-shaped feature.

In the case of the CMR presented by Lieder et al. 2012), the ACSVCS does not reach galaxies as faint as that study $\left(M_{\mathrm{V}} \sim-8.5\right.$ mag; using table $3(\mathrm{~m})$ in Fukugita et al. 1995), this translates into $\left.M_{\mathrm{g}} \sim-8.1 \mathrm{mag}\right)$. It might be expected that at the very low-mass end, the relation vanishes due to the physical limit of the metallicity of a galaxy. Here, age differences also become a relevant effect in producing the observed scatter. In addition, in these faint luminosities we are faced with large photometric and member identifica- tion errors. However, it should be noticed that in their figure 5, all but three of the spectroscopically confirmed Virgo members considered in the analysis, seem to define a quite linear trend spanning $-20 \lesssim M_{\mathrm{V}} \lesssim-16 \mathrm{mag}$, which transforms into $-19.6 \lesssim M_{\mathrm{g}} \lesssim-15.6 \mathrm{mag}$. This range is quite similar to that covered by the linear trend of the ACSVCS sample.

It is interesting to compare the slopes of Virgo's CMR with those found for other clusters in the literature. To this aim, and under the hypothesis that early-type galaxies colors are mainly dominated by old stellar populations, we can use several color-color relations presented in different papers.

In Virgo we have found $\Delta(g-z) / \Delta g \sim$ -0.09 . From the color-color relations presented by Forte et al. 2013) in their table 4, this translates into $\Delta\left(C-T_{1}\right) / \Delta T_{1} \sim-0.11$. In the Antlia cluster, Smith Castelli et al. 2012 find $\Delta T_{1} / \Delta\left(C-T_{1}\right)=-14.2$ when only elliptical confirmed members that do not display cE morphologies are considered. The inverse slope gives $\Delta\left(C-T_{1}\right) / \Delta T_{1} \sim-0.07$.

In the Hydra cluster, Misgeld et al. 2009) find $\Delta(V-I) / \Delta V=-0.040$. Through eq. (2) and (3) of Smith Castelli et al. 2008a), this transforms into $\Delta\left(C-T_{1}\right) / \Delta T_{1} \sim-0.10$. A similar result is obtained for Centaurus as it displays $\Delta(V-I) / \Delta V=-0.042 \pm 0.001$ Misgeld et al. 2009). For Fornax, a shallower slope is found $(\Delta(V-I) / \Delta V=-0.033 \pm 0.004$, Misgeld et al. 2008) which translates into $\Delta\left(C-T_{1}\right) / \Delta T_{1} \sim$ -0.08 . Secker et al. 1997) find for Coma $\Delta(B-$ $R) / \Delta R=-0.056$ which becomes $\Delta\left(C-T_{1}\right) / \Delta T_{1} \sim$ -0.08 .

From the above analysis we obtain that Fornax, Antlia and Coma seem to present a similar slope, shallower than those of Virgo, Hydra and Centaurus, which agree among them. In this sense, it is interesting to note that Lieder et al. 2012 have found a linear CMR for Virgo with a slope $\Delta(V-I) / \Delta V=-0.045 \pm 0.007$. This value, compared with those obtained in the same photometric system for Hydra, Centaurus and Fornax, is, within the errors, similar to those of Hydra and Centaurus, but steeper than that of Fornax, in agreement with our findings. In addition, Virgo and Centaurus seem to show similar values for the zero point of the relation. 


\section{3. $\quad \mathrm{cE}$ galaxies}

The location of $\mathrm{cE}$ galaxies in the colormagnitude and $\left\langle\mu_{\text {eff }}\right\rangle$-luminosity diagrams of Virgo are in agreement with those of cE galaxies in the Antlia cluster. That is, they are the galaxies that display the reddest colors, smallest $r_{\text {eff }}$ and brightest $\left\langle\mu_{\text {eff }}\right\rangle$ for their luminosities in a sample of early-type galaxies. The behavior of cEs defining a trend displaced from that followed by $\mathrm{dEs}$ in the $\left\langle\mu_{\text {eff }}\right\rangle$-luminosity plot rather than extending the region occupied by luminous galaxies, is also consistent with that shown by Chilingarian et al. 2009) for $\mathrm{cE}$ galaxies found through the Virtual Observatory. In consequence, the color-magnitude and $\left\langle\mu_{\text {eff }}\right\rangle$-luminosity diagrams complement each other giving a photometric tool to identify cE candidates.

As there are more galaxies of this kind in Virgo than, for example, in Antlia, we wonder if it is possible to link the position of $\mathrm{cE}$ galaxies in the color-magnitude and $\left\langle\mu_{\text {eff }}\right\rangle$-luminosity diagrams with some of their features in order to give clues about their physical origin.

For example, those displaying the reddest colors are VCC 1192, VCC 1199 and VCC 1297. Among them, VCC 1199 and VCC 1297 show the smallest $r_{\text {eff }}$ of the cEs sample, while VCC 1192 shows a size more similar to those of $\mathrm{dE}$ galaxies. This might be taken as evidence that VCC 1199 and VCC 1297 have experienced a stronger interaction with their massive neighbors than VCC 1192, generating their compactness but not necessarily their present integrated colors, as the three galaxies are comparable in this sense. However, VCC 1192 and VCC 1199 are found, in projection, closer $(\sim 4$ arcmin) to M 49, than VCC 1297 to M 87 ( 7.5 arcmin). And the relative radial velocities of the formers to M $49\left(\sim 400 \mathrm{~km} \mathrm{~s}^{-1}\right)$ are larger than that of the latter to M $87\left(\sim 200 \mathrm{~km} \mathrm{~s}^{-1}\right)$.

In contrast, VCC 1627 and VCC 1871 display the bluest colors of the cEs sample. The $r_{\text {eff }}$ of VCC 1627 is smaller than that of VCC 1192, while that of VCC 1871 is larger. VCC 1627 is placed, in projection, at $\sim 10.5$ arcmin from M 89 with a relative radial velocity of $\sim 100 \mathrm{~km} \mathrm{~s}^{-1}$, and at $\sim 7.5$ arcmin from NGC 4550 with a relative velocity of $\sim 140 \mathrm{~km} \mathrm{~s}^{-1}$. VCC 1871 is found at $\sim 19$ arcmin from M 59 with a relative radial velocity of $\sim 180$ $\mathrm{km} \mathrm{s}^{-1}$.
Therefore, at the moment, it is difficult to realize if there is a relationship between the spatial locations of $\mathrm{cE}$ galaxies and their colors or mass distribution, and to try to explain their unusual features. There is growing evidence of these objects being the remnants of disrupted systems as a consequence of their interaction with massive galaxies (see, for example, Smith Castelli et al. 2008b; Chilingarian et al. 2009; Huxor et al.2011). Some of our findings could be pointing towards a link between the present colors of the objects and their progenitors, and between their degree of compactness and the eccentricity of the orbits around their brighter companions. However, a more detailed study is necessary before arriving at firm conclusions.

\subsection{The color-magnitude and $\left\langle\mu_{\text {eff }}\right\rangle-$ luminosity diagrams link}

There seems to be a clear connection between the zones identified in the CMD of Virgo with those observed in the $\left\langle\mu_{\text {eff }}\right\rangle$-luminosity one. While faint and intermediate early-type galaxies define linear trends in both diagrams, the brightest galaxies and cEs depart from them in both plots. The exception are the galaxies showing evidence of recent star formation that follow the locus of early-type dwarfs in the $\left\langle\mu_{\text {eff }}\right\rangle$-luminosity diagram but clearly depart from the linear region of the CMR. This fact shows that if low-mass galaxies showing recent star formation are included in an early-type galaxy sample only because of their morphology or structural parameters, can cause significant contamination.

If we consider that the CMR represents a massmetallicity relation, and the $\left\langle\mu_{\text {eff }}\right\rangle$-luminosity diagram provides structural information, from these plots we can detect the link between structural and chemical properties in early-type galaxies. For example, all the galaxies that define the linear region of the CMR, display $r_{\text {eff }}$ in a restricted range of $\sim 0.5-2.0 \mathrm{kpc}$ Smith Castelli et al. 2008a; Misgeld \& Hilker 2011), which results in an increase of their $\left\langle\mu_{\mathrm{eff}}\right\rangle$ with luminosity. On the contrary, the brightest and $\mathrm{cE}$ galaxies define the highest and lowest boundarys in $r_{\text {eff }}$, respectively, displaying no clear trends in the CMD: cE galaxies show $r_{\text {eff }}<0.5 \mathrm{kpc}$, and the brightest ones, display $r_{\text {eff }} \gtrsim 8 \mathrm{kpc}$.

It is interesting to note that the connection be- 
tween the different zones in the CMD and $\left\langle\mu_{\text {eff }}\right\rangle-$ luminosity plane are also seen in other galaxy clusters, but with a different level of definition. The linear regions of both diagrams are easily detected in several clusters. However, the bright break and the population of $\mathrm{cE}$ galaxies are revealed depending on the group or cluster. For example, in Antlia there seems to be a break in the bright regime of the $\left\langle\mu_{\text {eff }}\right\rangle$-luminosity diagram, but there is no apreciable break in the CMR. In addition, only two $\mathrm{cE}$ galaxies have been identified until today. In the Hydra cluster, only two of the four galaxies that seem to define the bright break in the CMR, define a clear departure from the general trend in the $\left\langle\mu_{\mathrm{eff}}\right\rangle$-luminosity plot, and there are no $\mathrm{cE}$ galaxies reported in this cluster.

Excluding $\mathrm{dE} / \mathrm{dIrr}$ transition objects, both diagrams seem to point towards different evolutionary paths for early-type galaxies. The brightest galaxies may mainly assemble via mergers and/or accretion of low-mass satellites while cEs might arise from the disruption of more massive systems. On the other hand, intermediate and low-mass early-type galaxies would form in situ and passively evolve in a way that encounters and mergers do not significantly alter their global evolution. The fact that some but not all CMRs show a bright break would be in agreement with the merger/accretion processes taking place at different times and/or levels in different clusters. In a similar way, disruption events will generate objects with different degrees of compactness depending on the progenitor, the strength of the interaction and/or the stage in time of the disruption process. A mainly passive evolution would produce well defined and ordered relations.

This picture, and that of central regions of galaxies following the linear trend of the CMR, fits quite well in the galaxy formation scenarios analyzed by Oser et al. (2010) with numerical simulations. In these models, high-mass galaxies $\left(M_{\star} \lesssim 1.7 \times 10^{11} M_{\odot} h^{-1}\right)$ assemble mainly by accretion and merging, with about $80 \%$ of the present-day stars added by these events and building a more extended component, while low mass galaxies $\left(M_{\star} \lesssim 9 \times 10^{10} M_{\odot} h^{-1}\right)$ form up to $60 \%$ of their stellar mass in situ. These authors find that at high redshift, the assembly of galaxies of all masses is dominated by in situ star formation fed by cold flows. After this, the importance of stellar accretion increases with galaxy mass and, for the most massive galaxies, the accretion process is an important event at low redshifts.

The very faint end of both diagrams represents a more difficult task for interpretation because photometic errors, morphological missclassification, background contamination or, even, isophotal truncation (see figure 3 in Smith Castelli et al. 2012) can blur intrinsic trends. Before proposing an hypothesis for their origin in an observational basis, an effort is needed to spectroscopically confirm more low-mass cluster members. In this sense, it is worth noticing that, in the literature, CMDs of galaxy clusters are built with confirmed members up to $M_{\mathrm{V}} \sim-13 \mathrm{mag}$ as most Mieske et al. 2007; Misgeld et al. 2008, 2009). The alleged faint break in the Virgo cluster is not observed at this level for confirmed members. Only faint galaxy candidates seem to define such a break. In addition, De Rijcke et al, 2009) have built the CMD of $\mathrm{dE} / \mathrm{dSph}$ of different groups and clusters in a range of $8 \mathrm{mag}$, up to $M_{\mathrm{V}} \sim-10$ mag, and all follow the same tight linear relation extrapolated from that defined by the brightest galaxies.

\section{Conclusions}

In order to deepen our understanding of the CMR of early-type galaxies, we revisit the CMR of the Virgo cluster by analyzing that obtained by F06, and performing our own photometry of the 100 galaxies included in the ACSVCS. Although we obtain integrated colors and magnitudes with a simpler approach, we recover the general trend of the CMR reported by F06, except at the very bright end due to the surface brightness limit adopted for the spatially incomplete galaxies.

We have found that when galaxies displaying the highest luminosities, compact morphologies or evidence of recent star formation are excluded from the sample, a linear trend is recovered with a gap at $M_{g_{0}} \sim-17.5 \mathrm{mag}$, which is probably due to incompleteness effects. Therefore, it is not necessary to fit quadratic or higher order functions to the CMR of the Virgo cluster in the magnitude range $-20.5 \lesssim M_{g_{0}} \lesssim-16.5$ mag. Furthermore, the linear trend in this luminosity range is strong enough to be recovered with different color combinations and simple photometric methods, even 
considering a lesser number of galaxies .

Other Virgo CMRs reported in the literature are consistent with our analysis once a detailed morphological classification and/or membership identification is taken into account. Also, the slope of the CMR of Virgo has been compared with those of other galaxy clusters. Within the errors and considering the difficulty of comparing quantities obtained in different photometric systems, our results are also consistent with those of $\mathrm{Hy}-$ dra, and Centaurus, and it is steeper than those of Fornax, Antlia and Coma, which present similar slopes.

It is remarkable that the central regions of the brightest galaxies follow the linear trend depicted by the rest of the galaxies, rather than defining a stochastic distribution in the CMD due to their underestimated brightnesses. This finding might suport the picture in which large and massive elliptical galaxies observed today, are formed from compact and massive spheroids that enlarge their sizes through mergers and/or accretion of lowmass satellites.

We have identified four $\mathrm{cE}$ galaxies besides the well known VCC 1297 (NGC 4486B). Some of these galaxies have been previously reported as very compact and round systems, but we emphasize their similarity with cEs detected in other groups and clusters. They occupy very specific regions both in the color-magnitude and $\left\langle\mu_{\text {eff }}\right\rangle-$ luminosity diagrams: they are the reddest and most compact objects for their luminosities in a sample of early-type galaxies. In this sense, both diagrams analyzed together might be used to detect $\mathrm{cE}$ galaxy candidates.

A clear connection can be made between the different regions identified in the color-magnitude and $\left\langle\mu_{\text {eff }}\right\rangle$-luminosity diagrams of Virgo. The galaxies that define the bright break in the CMR, also detach from the rest of the systems in the $\left\langle\mu_{\text {eff }}\right\rangle$-luminosity diagram; the galaxies that follow the linear trend of the CMR also define a locus around a constant $r_{\text {eff }} \sim 1 \mathrm{kpc}$, displaying $r_{\text {eff }}$ between 0.5 and $2.0 \mathrm{kpc}$; and the $\mathrm{cE}$ galaxies which define a color limit for their luminosities in the CMD, also define $\left\langle\mu_{\text {eff }}\right\rangle$ and $r_{\text {eff }}$ limits in the $\left\langle\mu_{\text {eff }}\right\rangle$-luminosity plot.

The connection between the different zones occupied by different early-type galaxy sub-samples in the color-magnitude and $\left\langle\mu_{\text {eff }}\right\rangle$-luminosity diagrams could be taken as the manifestation of the link between color (abundance) and structural properties. In addition, it might be considered as evidence of the relative importance played by different formation mechanisms in different subsamples of early-type galaxies. Object disruption seems to be an important process behind cEs' evolution while mergers and/or satellite accretion might be the main drivers in the case of the very bright early-type galaxies. As the final products of such phenomena depend on, for example, the type and/or relative masses of the progenitors, it would be expected that they translate into no defined trends in both diagrams. On the contrary, a more passive evolution in which interactions play a less important role, might dominate the evolutionary path of intermediate and low-mass early-type galaxies producing well defined relations along an important range of masses.

If this scenario was correct, passive evolution would be a quite common process as the linear trends in the CMD and $\left\langle\mu_{\text {eff }}\right\rangle$-luminosity planes are observed in different groups and clusters. In this context, if the differences found in the slopes and zero points of some CMRs were significant, they could be interpreted as the manifestation of this process taking place with, for example, different enrichment rates or/and efficiencies in distinct associations.

The exception are the blue galaxies, which depart from the linear region of the CMR but follow the locus of normal early-type galaxies in the $\left\langle\mu_{\text {eff }}\right\rangle$-luminosity diagram. In this sense it should be noticed that their asymptotic colors derived from their color profiles $((g-z) \sim 1 \mathrm{mag})$ would locate them in the blue edge of the linear trend of the CMR for their luminosities. This might be pointing towards $\mathrm{dE} / \mathrm{dIrr}$ galaxies as previous stages of $\mathrm{dE}$ galaxies which define the blue border of the CMR, or as objects on their way towards the passive CMR. However, it is necessary to spectroscopically confirm more low-mass cluster members before being able to propose a solid hypothesis about their origin on an observational basis.

We thank the anonymous referee for her/his detailed revision and useful analysis of our paper which helped to greatly improve its content. We 
also thank Sergio Cellone for his help with the organization of the images, color maps and profiles of the galaxies in the webpage of our group, as well as for a fruitful discussion of the manuscript. All of the data presented in this paper were obtained from the Mikulski Archive for Space Telescopes (MAST). STScI is operated by the Association of Universities for Research in Astronomy, Inc., under NASA contract NAS5-26555. Support for MAST for non-HST data is provided by the NASA Office of Space Science via grant NNX09AF08G and by other grants and contracts. This work was funded with grants from Agencia de Promoción Científica y Tecnológica of Argentina (BID AR PICT 2010-0410), Consejo Nacional de Investigaciones Científicas y Técnicas de la República Argentina (PIP-11220080100712, PIP-11220080101611) and Facultad de Ciencias Astronómicas y Geofísicas (Universidad Nacional de La Plata, Argentina).

\section{REFERENCES}

Andreon, S., Cuillandre, J.-C., Puddu, E. \& Mellier, Y., 2006, MNRAS, 372, 60

Baum, W., 1959, PASP, 71, 106

Binggeli, B., \& Jerjen, H., 1998, A\&A, 333, 17

Chen, Ch.-W., Ct, P., West, A., Peng, E., \& Ferrarese, L., 2010, ApJS, 191, 1 (Ch10)

Chiboucas, K., Tully, B., Marzke, R., et al., ApJ, 723,251

Chilingarian, I., Cayatte, V., Revaz, Y., et al., 2009, Science, 326, 1379

Clemens, M., Panuzzo, P., Rampazzo, R., Vega, O., \& Bressan, A., 2011, MNRAS, 412, 2063

Côté, P., Marzke, R., West, M., \& Minniti, D., 2000, ApJ, 533, 869

Côté, P., Blakeslee, J., Ferrarese, L., et al., 2004, ApJS, 153, 223

De Rijcke, S., Penny, S., Conselice, C., Valcke, S., \& Held, E., 2009, MNRAS, 393, 798

Faber, S., 1973, ApJ, 179, 731

Ferrarese, L., Côté, P., Jordán, A., et al., 2006, ApJS, 164, 334 (F06)
Forte, J. C., Vega, E., \& Faifer, F., 2009, MNRAS, 397,1003

Forte, J. C., Faifer, F., Vega, E., et al., 2013, MNRAS, 431, 1405

Fukugita, M., Shimasaku, K., \& Ichikawa, T., 1995, PASP, 107, 945

Hilker, M., Infante, L., \& Richtler, T., 1999, A\&AS, 138,55

Hilz, M., Naab, T., Ostriker, J., et al., 2012, MNRAS, 425, 3119

Huxor, A., Phillipps, S., Price, J., \& Harniman, R., 2011, MNRAS, 414, 3557

Janz, L., \& Lisker, T., 2009, ApJ, 696, L102

Jiménez, N., Cora, S., Bassino, L., Tecce, T., \& Smith Castelli, A., 2011, MNRAS, 417, 785

Koekemoer, A., Fruchter, A., Hook, R. \& Hack, W., 2002, in The 2002 HST Calibration Workshop, ed. S. Arribas, A. Koekemoer, \& B. Whitmore, (Baltimore, MD: Space Telescope Science Institute), p.337

Lieder, S., Lisker, T., Hilker, M., Misgeld, I., Durrell, P., 2012, A\&A, 538, A69

López-Cruz, O., Barkhouse, W., \& Yee, H., 2004, ApJ, 614, 679

Mei, S., Blakeslee, J., Côté, P., et al., 2007, ApJ, 655,144

Mieske, S., Hilker, M., Infante, L., \& Mendes de Oliveira, C., 2007, A\&A, 463, 503

Misgeld, I., Mieske, S., \& Hilker, M., 2008, A\&A, 486,697

Misgeld, I., Hilker, M., \& Mieske, S., 2009, A\&A, 496,683

Misgeld, I., \& Hilker, M., 2011, MNRAS, 414, 3699

Oser, L., Ostriker, J., Naab, T., Johansson, P., \& Burkert, A., 2010, ApJ, 725, 2312

Sandage, A., 1972, ApJ, 176, 21

Secker, J., Harris, W., \& Plummer, J., 1997, PASP, 109, 1377 
Sirianni, M., Jee, M., Benítez, N., et al., 2005, PASP, 117, 1049

Smith Castelli, A., Bassino, L., Richtler, T., et al., 2008a, MNRAS, 386, 2311

Smith Castelli, A., Faifer, F., Richtler, T., \& Bassino, L., 2008b, MNRAS, 391, 685

Smith Castelli, A., Cellone, S., Faifer, F., et al., 2012, MNRAS, 419, 2472

Terlevich, A., Kuntschner, H., Bower, R., Caldwell, N., \& Sharples, R., 1999, MNRAS, 310, 445

Terlevich, A., Caldwell, N., \& Bower, R., 2001, MNRAS, 326, 1547

Trager, S., Faber, S., Worthey, G., \& Jesús González, J., 2000, AJ, 120, 165

Trujillo, I., Graham, A., \& Caon, N., 2001, MNRAS, 326, 869

Visvanathan, N. \& Sandage, A., 1977, ApJ, 216, 214

This 2-column preprint was prepared with the AAS LATEX macros v5.2. 
TABLE 1

OUR PHOtometry of the 100 galaxies INCLUded in the ACSVCS.

\begin{tabular}{|c|c|c|c|c|c|c|c|}
\hline & $\mathrm{VCC}$ & $\begin{array}{c}g \\
(m a g)\end{array}$ & $\begin{array}{l}(g-z) \\
(m a g)\end{array}$ & $\begin{array}{c}r_{\mathrm{eff}} \\
(\text { arcsec) }\end{array}$ & $\begin{array}{c}\left\langle\mu_{\mathrm{efff}}\right\rangle_{g} \\
\left(\operatorname{mag}^{-2 r c s e c}{ }^{-2}\right)\end{array}$ & $\epsilon$ & $\begin{array}{c}\theta \\
\text { (degrees) }\end{array}$ \\
\hline 1 & 9 & $14.05 \pm 0.01$ & $1.07 \pm 0.02$ & 25.51 & 23.08 & 0.20 & 50 \\
\hline 2 & 21 & $14.83 \pm 0.02$ & $0.88 \pm 0.03$ & 9.02 & 21.61 & 0.30 & -30 \\
\hline 3 & 33 & $15.07 \pm 0.02$ & $1.13 \pm 0.03$ & 8.41 & 21.69 & 0.20 & 15 \\
\hline 4 & 140 & $14.15 \pm 0.02$ & $1.20 \pm 0.02$ & 8.78 & 20.86 & 0.10 & 45 \\
\hline 5 & 200 & $14.89 \pm 0.01$ & $1.17 \pm 0.02$ & 11.48 & 22.18 & 0.10 & 9 \\
\hline 6 & 230 & $15.60 \pm 0.02$ & $1.17 \pm 0.03$ & 8.55 & 22.26 & 0.10 & -45 \\
\hline 7 & 355 & $12.22 \pm 0.01$ & $1.50 \pm 0.01$ & 7.15 & 18.49 & 0.10 & 45 \\
\hline 8 & 369 & $11.93 \pm 0.01$ & $1.53 \pm 0.01$ & $\ldots$ & $\ldots$ & 0.10 & 9 \\
\hline 9 & 437 & $14.17 \pm 0.02$ & $1.25 \pm 0.02$ & 17.42 & 22.37 & 0.30 & -35 \\
\hline 10 & 538 & $16.02 \pm 0.02$ & $1.15 \pm 0.02$ & 4.37 & 21.22 & 0.10 & -4 \\
\hline 11 & 543 & $14.38 \pm 0.01$ & $1.20 \pm 0.02$ & 14.54 & 22.19 & 0.40 & 45 \\
\hline 12 & 571 & $15.13 \pm 0.02$ & $1.10 \pm 0.03$ & 9.45 & 22.01 & 0.40 & -4 \\
\hline 13 & 575 & $13.91 \pm 0.03$ & $1.33 \pm 0.04$ & 5.48 & 19.59 & 0.10 & -4 \\
\hline 14 & 654 & $12.08 \pm 0.03$ & $1.44 \pm 0.03$ & $\ldots$ & $\ldots$ & 0.30 & -15 \\
\hline 15 & 685 & $11.69 \pm 0.05$ & $1.49 \pm 0.07$ & 11.68 & 19.03 & 0.30 & 90 \\
\hline 16 & 698 & $13.25 \pm 0.03$ & $1.31 \pm 0.04$ & 13.14 & 20.84 & 0.30 & -30 \\
\hline 17 & 731 & $10.90 \pm 0.01$ & $1.54 \pm 0.01$ & $\ldots$ & $\ldots$ & 0.30 & 90 \\
\hline 18 & 751 & $14.70 \pm 0.02$ & $1.27 \pm 0.03$ & 9.25 & 21.53 & 0.20 & -4 \\
\hline 19 & 759 & $11.64 \pm 0.02$ & $1.54 \pm 0.03$ & $\ldots$ & $\ldots$ & 0.40 & -25 \\
\hline 20 & 763 & $10.49 \pm 0.01$ & $1.56 \pm 0.01$ & $\ldots$ & $\ldots$ & 0.10 & 0 \\
\hline 21 & 778 & $12.33 \pm 0.02$ & $1.42 \pm 0.02$ & 8.40 & 18.94 & 0.30 & 45 \\
\hline 22 & 784 & $12.28 \pm 0.02$ & $1.42 \pm 0.02$ & 11.97 & 19.67 & 0.30 & 0 \\
\hline 23 & 798 & $10.58 \pm 0.02$ & $1.42 \pm 0.02$ & $\ldots$ & $\ldots$ & 0.30 & 90 \\
\hline 24 & 828 & $12.78 \pm 0.01$ & $1.46 \pm 0.02$ & 9.38 & 19.64 & 0.30 & 45 \\
\hline 25 & 856 & $14.19 \pm 0.02$ & $1.18 \pm 0.03$ & 14.51 & 22.00 & 0.20 & 9 \\
\hline 26 & 881 & $10.61 \pm 0.01$ & $1.53 \pm 0.01$ & $\ldots$ & $\ldots$ & 0.30 & -20 \\
\hline 27 & 944 & $11.97 \pm 0.02$ & $1.42 \pm 0.03$ & $\ldots$ & $\ldots$ & 0.50 & -65 \\
\hline 28 & 1025 & $12.70 \pm 0.01$ & $1.41 \pm 0.02$ & 9.42 & 19.57 & 0.20 & 40 \\
\hline 29 & 1030 & $11.55 \pm 0.02$ & $1.48 \pm 0.04$ & 12.70 & 19.06 & 0.20 & 59 \\
\hline 30 & 1049 & $14.93 \pm 0.02$ & $1.08 \pm 0.02$ & 7.07 & 21.17 & 0.10 & -45 \\
\hline 31 & 1062 & $11.18 \pm 0.02$ & $1.50 \pm 0.03$ & $\ldots$ & & 0.50 & -25 \\
\hline 32 & 1075 & $14.95 \pm 0.02$ & $1.14 \pm 0.03$ & 15.52 & 22.90 & 0.10 & -60 \\
\hline 33 & 1087 & $13.97 \pm 0.01$ & $1.25 \pm 0.01$ & 16.69 & 22.08 & 0.30 & 0 \\
\hline 34 & 1125 & $13.02 \pm 0.03$ & $1.33 \pm 0.05$ & $\ldots$ & & 0.60 & -80 \\
\hline 35 & 1146 & $12.76 \pm 0.02$ & $1.34 \pm 0.02$ & 13.33 & 20.38 & 0.15 & 45 \\
\hline 36 & 1154 & $11.35 \pm 0.01$ & $1.59 \pm 0.01$ & $\ldots$ & $\ldots$ & 0.20 & 2 \\
\hline 37 & 1178 & $13.14 \pm 0.02$ & $1.44 \pm 0.03$ & 5.82 & 18.96 & 0.15 & 59 \\
\hline 38 & 1185 & $15.56 \pm 0.02$ & $1.27 \pm 0.02$ & 14.13 & 23.31 & 0.10 & -45 \\
\hline 39 & 1192 & $14.90 \pm 0.02$ & $1.46 \pm 0.03$ & 4.73 & 20.26 & 0.05 & 0 \\
\hline 40 & 1199 & $16.44 \pm 0.02$ & $1.54 \pm 0.02$ & 2.09 & 20.03 & 0.10 & -4 \\
\hline
\end{tabular}


TABLE 1-Continued

\begin{tabular}{|c|c|c|c|c|c|c|c|}
\hline & $\mathrm{VCC}$ & $\begin{array}{c}g \\
(m a g)\end{array}$ & $\begin{array}{l}(g-z) \\
(m a g)\end{array}$ & $\begin{array}{c}r_{\mathrm{eff}_{\mathrm{g}}} \\
(\operatorname{arcsec})\end{array}$ & $\begin{array}{c}\left\langle\mu_{\mathrm{eff}}\right\rangle_{g} \\
\left(\operatorname{mag} \operatorname{arcsec}^{-2}\right)\end{array}$ & $\epsilon$ & $\begin{array}{c}\theta \\
(\text { degrees })\end{array}$ \\
\hline 41 & 1226 & $10.02 \pm 0.01$ & $1.57 \pm 0.01$ & $\cdots$ & $\cdots$ & 0.25 & 50 \\
\hline 42 & 1231 & $11.16 \pm 0.02$ & $1.50 \pm 0.02$ & $\ldots$ & $\ldots$ & 0.30 & -15 \\
\hline 43 & 1242 & $12.34 \pm 0.03$ & $1.39 \pm 0.04$ & 13.50 & 19.99 & 0.20 & 80 \\
\hline 44 & 1250 & $12.86 \pm 0.02$ & $1.29 \pm 0.03$ & 11.09 & 20.07 & 0.20 & 85 \\
\hline 45 & 1261 & $13.59 \pm 0.01$ & $1.19 \pm 0.02$ & 14.96 & 21.46 & 0.30 & 30 \\
\hline 46 & 1279 & $11.96 \pm 0.01$ & $1.44 \pm 0.01$ & 11.09 & 19.18 & 0.20 & 45 \\
\hline 47 & 1283 & $13.22 \pm 0.02$ & $1.42 \pm 0.03$ & 15.20 & 21.12 & 0.10 & 40 \\
\hline 48 & 1297 & $14.19 \pm 0.02$ & $1.52 \pm 0.02$ & 2.32 & 18.01 & 0.10 & 45 \\
\hline 49 & 1303 & $12.89 \pm 0.02$ & $1.41 \pm 0.02$ & 11.00 & 20.10 & 0.40 & -55 \\
\hline 50 & 1316 & $10.21 \pm 0.01$ & $1.59 \pm 0.01$ & $\ldots$ & $\ldots$ & 0.15 & 45 \\
\hline 51 & 1321 & $12.77 \pm 0.02$ & $1.30 \pm 0.02$ & 14.95 & 20.64 & 0.20 & 50 \\
\hline 52 & 1327 & $13.12 \pm 0.02$ & $1.52 \pm 0.03$ & 7.71 & 19.55 & 0.20 & 55 \\
\hline 53 & 1355 & $14.69 \pm 0.01$ & $1.21 \pm 0.03$ & 20.63 & 23.26 & 0.10 & 45 \\
\hline 54 & 1407 & $15.04 \pm 0.02$ & $1.19 \pm 0.01$ & 10.32 & 22.10 & 0.10 & 45 \\
\hline 55 & 1422 & $13.73 \pm 0.01$ & $1.19 \pm 0.01$ & 17.44 & 21.93 & 0.20 & 45 \\
\hline 56 & 1431 & $14.35 \pm 0.01$ & $1.37 \pm 0.01$ & 9.25 & 21.17 & 0.10 & 4 \\
\hline 57 & 1440 & $14.79 \pm 0.01$ & $1.17 \pm 0.02$ & 6.78 & 20.94 & 0.10 & -9 \\
\hline 58 & 1475 & $13.13 \pm 0.02$ & $1.29 \pm 0.03$ & 8.20 & 19.69 & 0.30 & 50 \\
\hline 59 & 1488 & $14.86 \pm 0.02$ & $0.91 \pm 0.02$ & 9.02 & 21.63 & 0.30 & -40 \\
\hline 60 & 1489 & $15.98 \pm 0.02$ & $1.06 \pm 0.03$ & 10.00 & 22.97 & 0.30 & -45 \\
\hline 61 & 1499 & $15.01 \pm 0.03$ & $0.76 \pm 0.04$ & 5.39 & 20.66 & 0.10 & -45 \\
\hline 62 & 1512 & $15.80 \pm 0.02$ & $1.16 \pm 0.03$ & 8.55 & 22.45 & 0.20 & -45 \\
\hline 63 & 1528 & $14.49 \pm 0.01$ & $1.23 \pm 0.01$ & 8.83 & 21.21 & 0.10 & 0 \\
\hline 64 & 1535 & $10.69 \pm 0.02$ & $1.56 \pm 0.03$ & $\ldots$ & $\ldots$ & 0.50 & 4 \\
\hline 65 & 1537 & $12.56 \pm 0.02$ & $1.43 \pm 0.03$ & 7.99 & 19.07 & 0.30 & 65 \\
\hline 66 & 1539 & $15.86 \pm 0.02$ & $1.22 \pm 0.02$ & 14.27 & 23.63 & 0.10 & 45 \\
\hline 67 & 1545 & $14.90 \pm 0.03$ & $1.26 \pm 0.04$ & 9.31 & 21.74 & 0.30 & -15 \\
\hline 68 & 1619 & $12.24 \pm 0.02$ & $1.39 \pm 0.02$ & 10.63 & 19.37 & 0.60 & 65 \\
\hline 69 & 1627 & $15.26 \pm 0.01$ & $1.41 \pm 0.01$ & 3.44 & 19.93 & 0.10 & 9 \\
\hline 70 & 1630 & $12.64 \pm 0.01$ & $1.50 \pm 0.02$ & 11.35 & 19.91 & 0.20 & -18 \\
\hline 71 & 1632 & $10.95 \pm 0.01$ & $1.59 \pm 0.01$ & $\ldots$ & $\ldots$ & 0.10 & 9 \\
\hline 72 & 1661 & $15.89 \pm 0.02$ & $1.18 \pm 0.03$ & 16.08 & 23.92 & 0.10 & 9 \\
\hline 73 & 1664 & $11.91 \pm 0.02$ & $1.48 \pm 0.03$ & $\ldots$ & $\ldots$ & 0.40 & -55 \\
\hline 74 & 1692 & $11.61 \pm 0.02$ & $1.48 \pm 0.03$ & $\ldots$ & $\ldots$ & 0.60 & 50 \\
\hline 75 & 1695 & $14.38 \pm 0.02$ & $1.12 \pm 0.02$ & 19.52 & 22.83 & 0.10 & -4 \\
\hline 76 & 1720 & $12.17 \pm 0.02$ & $1.42 \pm 0.03$ & 17.42 & 20.37 & 0.30 & 80 \\
\hline 77 & 1743 & $15.53 \pm 0.03$ & $1.05 \pm 0.05$ & 10.62 & 22.65 & 0.30 & 35 \\
\hline 78 & 1779 & $14.82 \pm 0.03$ & $1.00 \pm 0.04$ & 10.88 & 22.00 & 0.50 & -45 \\
\hline 79 & 1826 & $15.55 \pm 0.01$ & $1.13 \pm 0.02$ & 6.27 & 21.53 & 0.30 & 35 \\
\hline 80 & 1828 & $15.23 \pm 0.02$ & $1.15 \pm 0.03$ & 12.80 & 22.76 & 0.20 & 45 \\
\hline
\end{tabular}


TABLE 1-Continued

\begin{tabular}{cccccccc}
\hline \hline & VCC & $\begin{array}{c}g \\
(\mathrm{mag})\end{array}$ & $\begin{array}{c}(g-z) \\
(\mathrm{mag})\end{array}$ & $\begin{array}{c}r_{\mathrm{eff}_{\mathrm{g}}} \\
(\text { arcsec })\end{array}$ & $\begin{array}{c}\left\langle\mu_{\mathrm{eff}}\right\rangle_{g} \\
\left(\text { mag arcsec }^{-2}\right)\end{array}$ & $\epsilon$ & $\begin{array}{c}\theta \\
(\text { degrees })\end{array}$ \\
\hline 81 & 1833 & $14.53 \pm 0.02$ & $1.21 \pm 0.03$ & 7.07 & 20.77 & 0.10 & 0 \\
82 & 1857 & $15.01 \pm 0.02$ & $0.94 \pm 0.02$ & 19.17 & 23.42 & 0.40 & 0 \\
83 & 1861 & $14.20 \pm 0.01$ & $1.28 \pm 0.02$ & 15.94 & 22.21 & 0.10 & 45 \\
84 & 1871 & $14.19 \pm 0.02$ & $1.42 \pm 0.02$ & 6.41 & 20.22 & 0.10 & 4 \\
85 & 1883 & $12.07 \pm 0.01$ & $1.33 \pm 0.02$ & 14.35 & 19.85 & 0.30 & 80 \\
86 & 1886 & $15.37 \pm 0.01$ & $1.01 \pm 0.02$ & 12.26 & 22.81 & 0.40 & 65 \\
87 & 1895 & $14.99 \pm 0.02$ & $1.08 \pm 0.03$ & 9.29 & 21.83 & 0.50 & -60 \\
88 & 1903 & $10.94 \pm 0.01$ & $1.55 \pm 0.02$ & $\ldots$ & $\ldots$ & 0.30 & 45 \\
89 & 1910 & $14.24 \pm 0.01$ & $1.36 \pm 0.01$ & 11.25 & 21.49 & 0.10 & 45 \\
90 & 1913 & $13.05 \pm 0.03$ & $1.33 \pm 0.04$ & 13.17 & 20.65 & 0.50 & 45 \\
91 & 1938 & $11.95 \pm 0.03$ & $1.42 \pm 0.04$ & 9.43 & 18.82 & 0.30 & 45 \\
92 & 1948 & $15.47 \pm 0.02$ & $1.02 \pm 0.03$ & 10.82 & 22.64 & 0.40 & -20 \\
93 & 1978 & $10.25 \pm 0.01$ & $1.63 \pm 0.01$ & $\ldots$ & $\ldots$ & 0.20 & -9 \\
94 & 1993 & $15.48 \pm 0.02$ & $1.19 \pm 0.02$ & 9.44 & 22.35 & 0.10 & 4 \\
95 & 2000 & $11.94 \pm 0.03$ & $1.43 \pm 0.04$ & 8.55 & 18.60 & 0.20 & -9 \\
96 & 2019 & $14.43 \pm 0.01$ & $1.15 \pm 0.02$ & 14.55 & 22.24 & 0.20 & -80 \\
97 & 2048 & $14.00 \pm 0.01$ & $1.18 \pm 0.02$ & 10.08 & 21.01 & 0.50 & 65 \\
98 & 2050 & $15.22 \pm 0.03$ & $1.16 \pm 0.04$ & 10.52 & 22.33 & 0.20 & 85 \\
99 & 2092 & $11.39 \pm 0.02$ & $1.51 \pm 0.03$ & $\ldots$ & $\ldots$ & 0.40 & -80 \\
100 & 2095 & $11.69 \pm 0.04$ & $1.39 \pm 0.06$ & $\ldots$ & $\ldots$ & 0.40 & -15 \\
\hline
\end{tabular}

Note. - Col. (2) shows the VCC designation of the galaxies. Col. (3) shows not extinction corrected $g$ magnitudes. Col. (4) lists not reddening corrected $(g-z)$ colors. Col. (5) shows $r_{\text {eff }}$ obtained in this work in the $g$ band for galaxies that are fully contained in the ACS FOV. Col. (6) lists the corresponding $\left\langle\mu_{\text {eff }}\right\rangle$ in the $g$ band. Cols. (7) and (8) show the fixed ellipticity and position angle used to obtain brightness and color profiles from which integrated magnitudes and colors were calculated. 
TABLE 2

RESUlts OF LEAST-SQUARE FITS PERFORMED TO THE LINEAR REGION OF THE CMR OF EARLY-TYPE GALAXIES IN THE ViRgo CLUSTER

\begin{tabular}{|c|c|c|c|c|c|}
\hline Sample & Data & $a$ & $b$ & $r$ & $\sigma_{(g-z)_{0}}$ \\
\hline \multicolumn{6}{|c|}{ 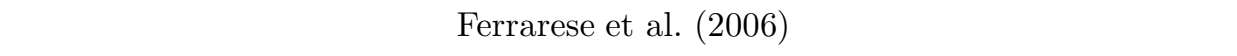 } \\
\hline Whole trend & 74 & $1.28 \pm 0.010$ & $-0.097 \pm 0.008$ & -0.84 & 0.06 \\
\hline Bright region & 35 & $1.31 \pm 0.026$ & $-0.069 \pm 0.020$ & -0.58 & 0.07 \\
\hline Faint region & 39 & $1.34 \pm 0.021$ & $-0.163 \pm 0.017$ & -0.47 & 0.10 \\
\hline Reduced sample & 61 & $1.28 \pm 0.012$ & $-0.098 \pm 0.011$ & -0.79 & 0.06 \\
\hline Bright Reduced sample & 25 & $1.34 \pm 0.035$ & $-0.034 \pm 0.036$ & -0.46 & 0.09 \\
\hline Faint Reduced sample & 36 & $1.23 \pm 0.025$ & $-0.087 \pm 0.017$ & -0.49 & 0.04 \\
\hline \multicolumn{6}{|c|}{ Our work } \\
\hline Whole trend & 64 & $1.27 \pm 0.011$ & $-0.089 \pm 0.009$ & -0.83 & 0.07 \\
\hline Bright region & 26 & $1.28 \pm 0.025$ & $-0.094 \pm 0.029$ & -0.51 & 0.06 \\
\hline Faint region & 38 & $1.21 \pm 0.034$ & $-0.046 \pm 0.023$ & -0.35 & 0.08 \\
\hline Reduced sample & 61 & $1.27 \pm 0.012$ & $-0.089 \pm 0.009$ & -0.83 & 0.07 \\
\hline Bright Reduced sample & 24 & $1.28 \pm 0.025$ & $-0.101 \pm 0.030$ & -0.55 & 0.06 \\
\hline Faint Reduced sample & 37 & $1.21 \pm 0.034$ & $-0.047 \pm 0.024$ & -0.38 & 0.07 \\
\hline \multicolumn{6}{|c|}{ Chen et al. (2010) } \\
\hline Whole trend & 79 & $1.27 \pm 0.012$ & $-0.078 \pm 0.008$ & -0.81 & 0.07 \\
\hline Bright region & 42 & $1.28 \pm 0.024$ & $-0.070 \pm 0.015$ & -0.64 & 0.05 \\
\hline Faint region & 37 & $1.19 \pm 0.033$ & $-0.032 \pm 0.026$ & -0.31 & 0.13 \\
\hline Reduced sample & 61 & $1.27 \pm 0.011$ & $-0.082 \pm 0.010$ & -0.74 & 0.07 \\
\hline Bright Reduced sample & 25 & $1.28 \pm 0.033$ & $-0.066 \pm 0.032$ & -0.52 & 0.08 \\
\hline Faint Reduced sample & 36 & $1.19 \pm 0.034$ & $-0.033 \pm 0.026$ & -0.31 & 0.06 \\
\hline
\end{tabular}

Note. - The fitted function is of the form $(g-z)_{0}=a+b\left(M_{g_{0}}+18\right)$. Col. (1) indicates the different samples considered to perform the fits. Col. (2) gives the number of data points. Col. (3) and (4) list the slopes and zero-points of the reddening and extinction corrected mean linear CMRs. Col. (5) shows the Pearson correlation coefficient, $r$, of the different fits. Col. (6) presents the intrinsic color scatter which was obtained as in Smith Castelli et al. 2012). The adopted limiting magnitude to separate the bright and faint regions of the linear trends is that of the observed intermediate gap $\left(M_{g_{0}} \sim-17.5 \mathrm{mag}\right)$. 


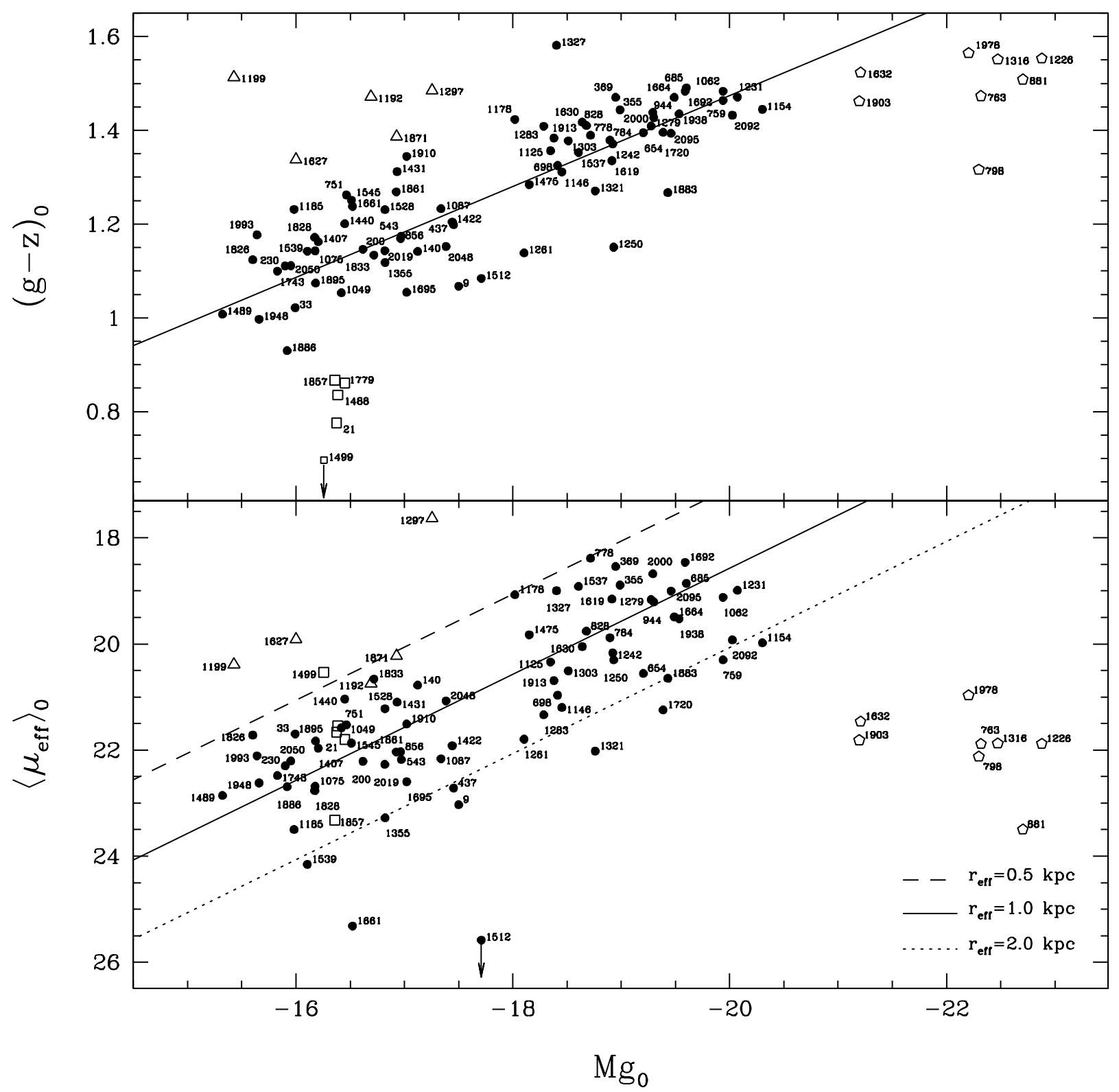

Fig. 1.-Distance, extinction and reddening corrected color-magnitude (top) and $\left\langle\mu_{\mathrm{eff}}\right\rangle-$ magnitude (bottom) diagrams of the galaxies included in the ACSVCS, built from magnitudes, colors and $\left\langle\mu_{\mathrm{eff}}\right\rangle$ values reported by F06. The numbers correspond to the VCC designation of each galaxy. With open pentagons, we show the galaxies that define a break in the CMR towards the bright end. Open triangles depict objects consistent with being $\mathrm{cE}$ galaxies, and open squares, those showing blue centers and evidence of recent star formation. For clarity, in the bottom panel, we have not labeled VCC 1488 and VCC 1779, two galaxies displaying blue centers. The dashed line in the top panel represents the linear fit to the CMR when bright ellipticals, $\mathrm{cE}$ galaxies and dwarfs with evidence of recent star formation are excluded from the sample. The different lines shown in the bottom panel depict loci of constant $r_{\text {eff }}$. 


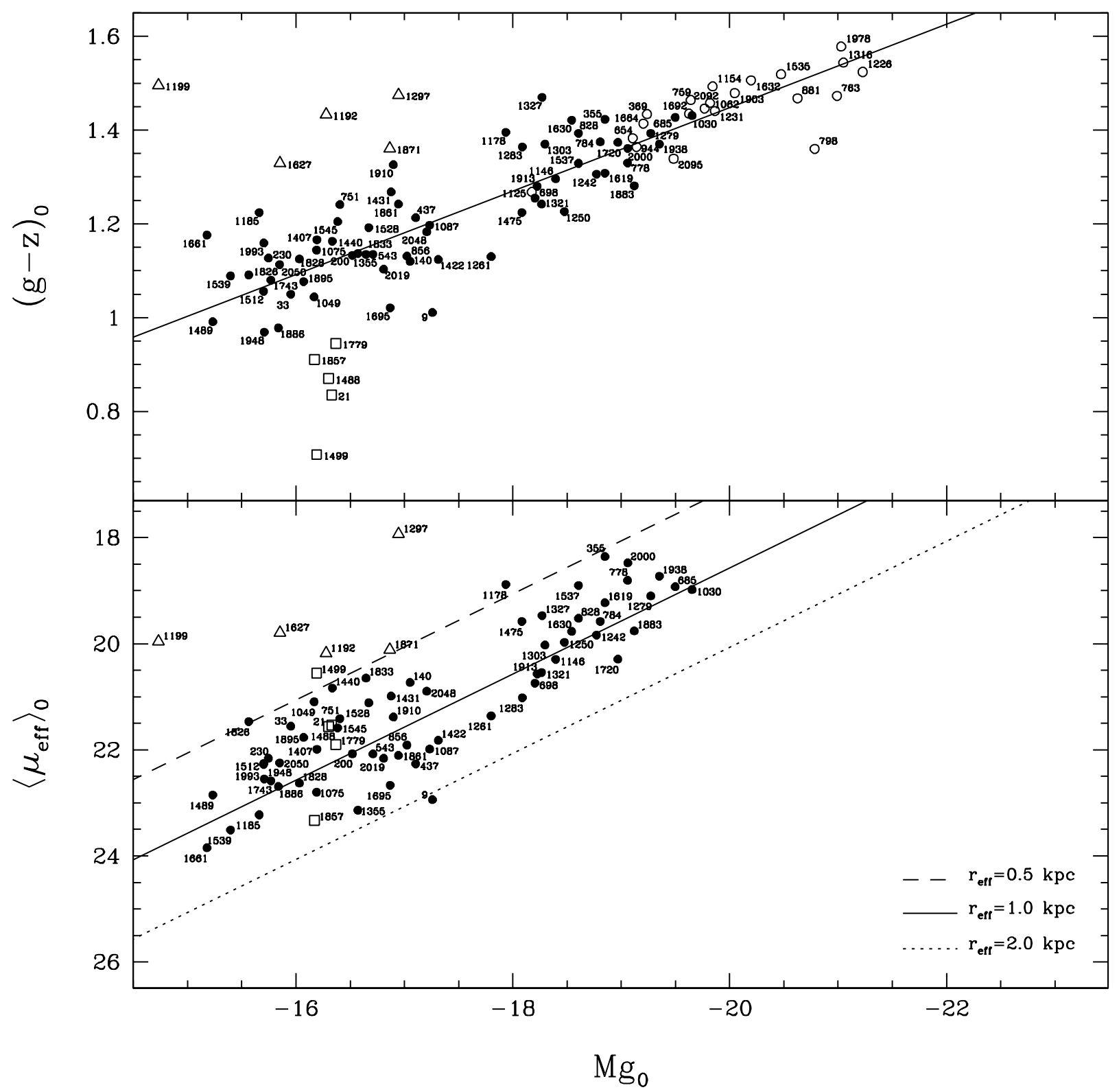

Fig. 2.-Distance, extinction and reddening corrected color-magnitude (top) and $\left\langle\mu_{\mathrm{eff}}\right\rangle$-magnitude (bottom) diagrams of the galaxies included in the ACSVCS, built from our photometry. The numbers correspond to the VCC designation of each galaxy. In the top panel, we depict with open circles incomplete galaxies, not shown in the bottom panel as we were not able to estimate their $r_{\text {eff }}$. With open triangles we show the objects consistent with being $\mathrm{cE}$ galaxies, and with open squares those that show blue centers and evidence of recent star formation. The dashed line in the top panel represents the linear fit to the CMR when incomplete galaxies, cEs and dwarfs with evidence of recent star formation are excluded from the sample. The different lines shown in the bottom panel depict loci of constant $r_{\mathrm{eff}}$. 

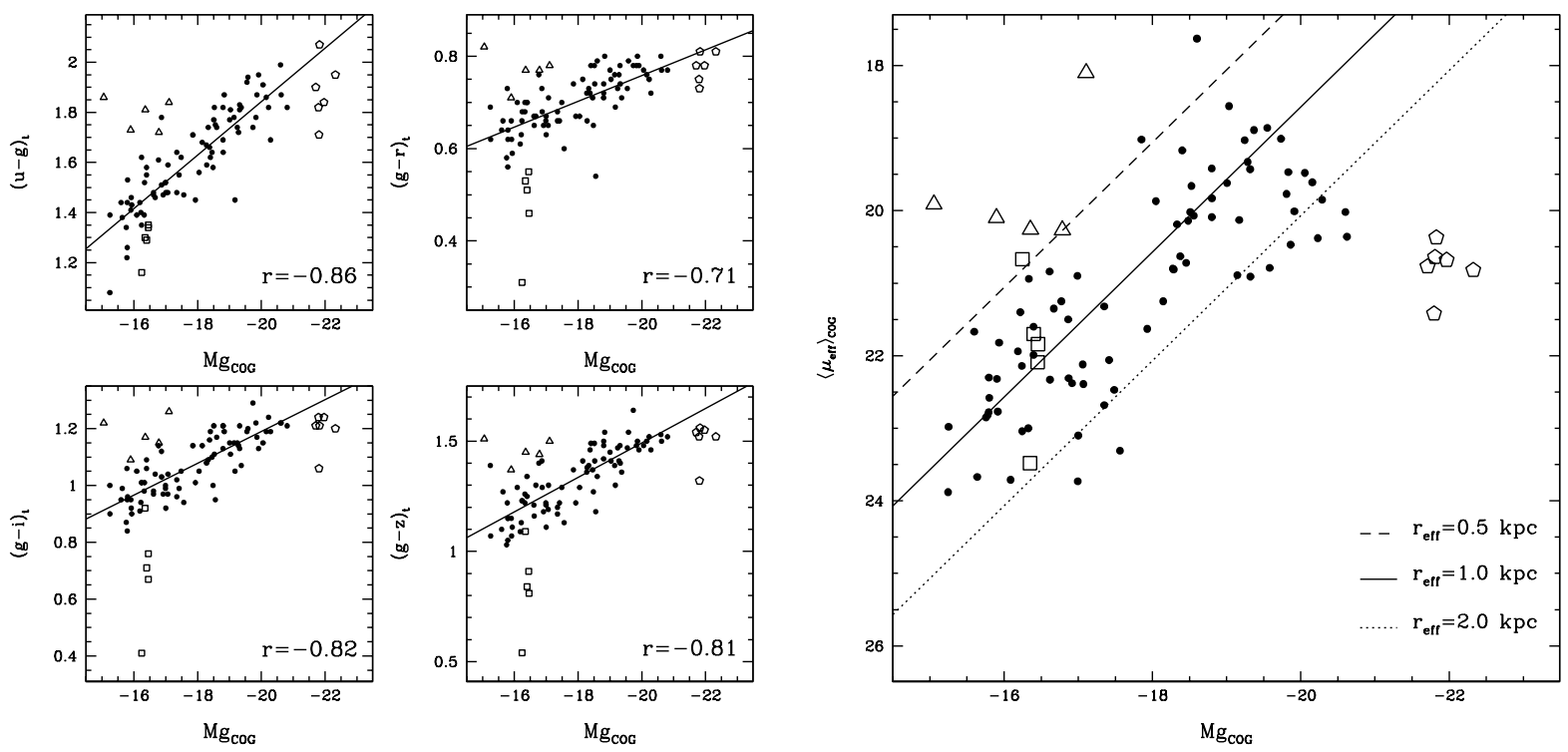

Fig. 3.- Left: Extinction and reddening uncorrected CMRs obtained from the SDSS photometry of Ch10. Symbols code is identical to that of Figure 1 The solid lines represent the linear fits performed to early-type galaxies, excluding those of the bright break, $\mathrm{cE}$ galaxies and objects with evidence of recent star formation. The Pearson correlation coefficient $r$ found for each fit is shown in each panel. Right: $\left\langle\mu_{\mathrm{eff}}\right\rangle$-magnitude diagram built from the data of Ch10. The different lines depict loci of constant $r_{\mathrm{eff}}$. 


\section{A. Our values compared with those of F06}

In Figure 4 we present three plots that show, as a function of luminosity, the differences between our $g$ magnitudes, $(g-z)$ colors and $r_{\text {eff }}$ values, and those found by F06 $\left(\Delta M_{g}=M_{g}-M_{g_{F 06}}, \Delta(g-z)=\right.$ $(g-z)-(g-z)_{F 06}$ and $\Delta r_{\mathrm{eff}}=r_{\mathrm{eff}}-r_{\mathrm{eff}_{F 06}}$, respectively). We can see that our magnitudes tend to be fainter, our colors bluer and our $r_{\text {eff }}$ values smaller than those of F06. This is consistent with the fact that we have isophotal limited brightnesses while those of F06 are obtained from the integration to infinity of theoretic brightness profiles. In addition, it is expected that our colors are bluer than those of F06 as early-type galaxies tend to be redder in their central regions, becoming bluer outwards. Our colors are calculated as the difference between the integrated magnitudes obtained from the integration of the observed brightness profiles. F06 measured their colors between $1^{\prime \prime}$ and $1 r_{\text {eff }}$.

In particular, for complete galaxies, we obtain $\left\langle\Delta M_{g}\right\rangle=0.13$ mag excluding the four more separated galaxies, $\langle\Delta(g-z)\rangle=-0.03$ mag excluding the two more separated objects, and $\left\langle\Delta r_{\text {eff }}\right\rangle=-1.53^{\prime \prime}$ excluding the six more separated values.

The most important deviation in color is shown by VCC $1499(\langle\Delta(g-z)\rangle=0.25 \mathrm{mag})$. VCC 1499 display a strong gradient within $1 r_{\text {eff }}$. Therefore, this discrepancy probably arises because F06 exclude the external (and reddest) regions of the galaxy to obtain its color.

The largest deviations both in luminosity $\left(\Delta M_{g}=2.01 \mathrm{mag}\right)$ and $r_{\text {eff }}\left(\Delta r_{\text {eff }}=-488.95^{\prime \prime}\right)$ correspond to VCC 1512, followed by those of VCC $1661\left(\Delta M_{g}=1.34 \mathrm{mag}\right.$ and $\left.\Delta r_{\text {eff }}=-42.42^{\prime \prime}\right)$. The case of VCC 1512 was already noticed in Section 3.1 and is related to unreliable quantities as reported by F06. In the case of VCC 1661, the $g$ brightness profile reaches $\mu_{g_{0}} \approx 27 \mathrm{mag} \operatorname{arcsec}^{-2}$ at $r \approx 40^{\prime \prime}$. Therefore, the value of $r_{\text {eff }}=58.50^{\prime \prime}$ reported by F06 is clearly overestimated, giving also an overestimated luminosity. VCC 1539 presents a similar situation though not so severe, as F06 reported $r_{\text {eff }}=26.53^{\prime \prime}$, but its brightness profile reaches $\mu_{g_{0}} \approx 27 \mathrm{mag} \operatorname{arcsec}^{-2}$ at $r \approx 30^{\prime \prime}$.

VCC 1321, VCC 1720 and VCC 1883 show significant differences in $r_{\text {eff }}$ but not in integrated magnitudes. This situation clearly points towards a discrepancy arising because of the different methods used to obtain $r_{\text {eff }}$. It is difficult to say if the fitting method overestimates the values, or the isophotal limited analysis underestimate them. This would deserve an analysis that it is beyond the scope of this paper. However, it can be noticed that in the case of VCC 1883, we have obtained a brightness profile that would prevent a good fit even considering two components.

We also point out that when we measure integrated colors in the same way as F06 do (that is, integrating the brightness profiles between $1^{\prime \prime}$ and $\left.1 r_{\text {eff }}\right)$, we obtain $\left\langle(g-z)_{r e f f}-(g-z)_{F 06}\right\rangle=0.009 \pm 0.03$ mag. 


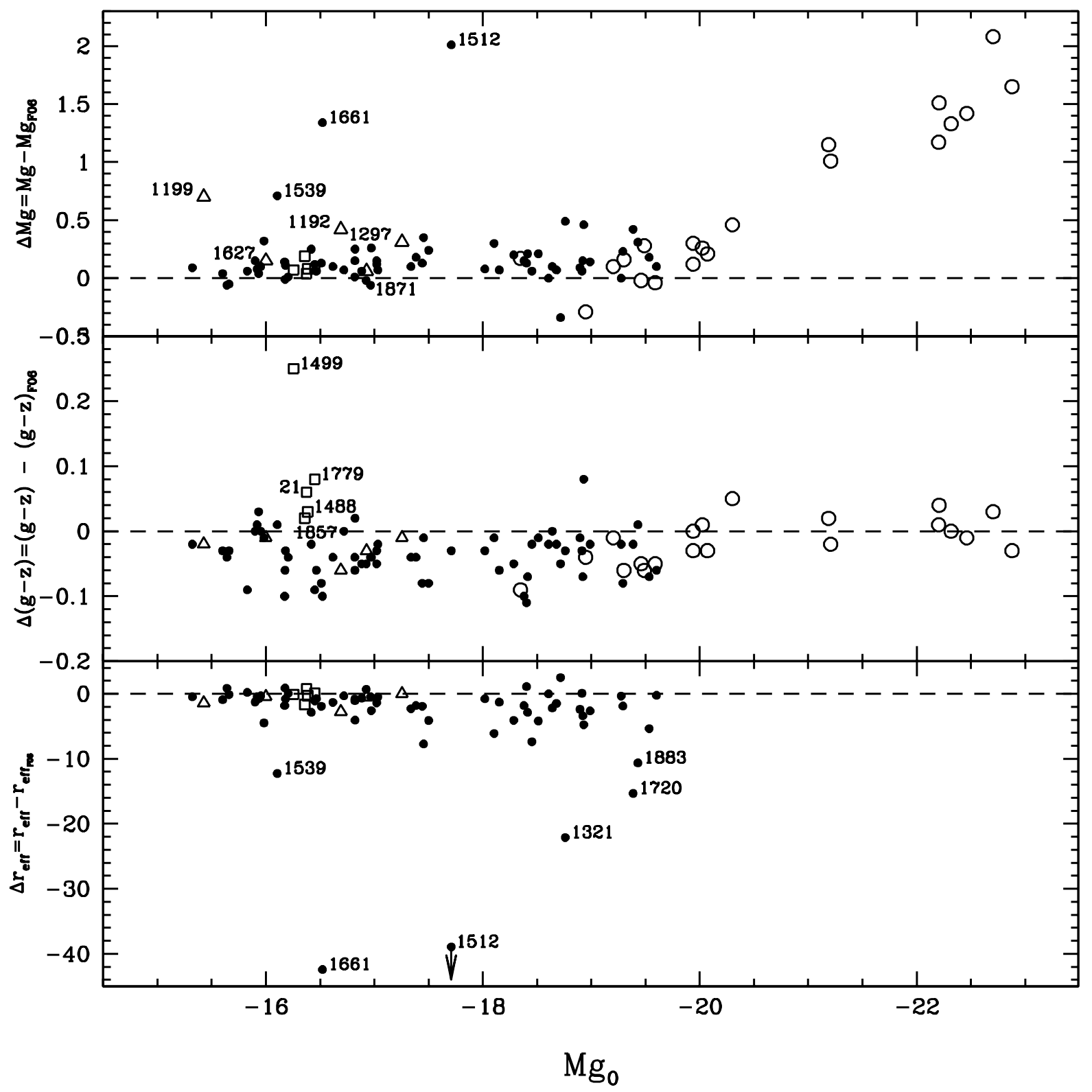

Fig. 4.- We show the differences between our $g$ magnitudes (top), $(g-z)$ colors (center) and $r_{\text {eff }}$ values in $\operatorname{arcsec}$ (bottom), and those reported by F06 for the galaxies of the ACSVCS. We depict with open circles incomplete galaxies. For clarity, in the top plot we only label the $\mathrm{cE}$ galaxies (open triangles) and in the centered one, the blue galaxies (open squares). In all the plots we also label the most discrepant galaxies that are complete within the ACS frames. 